\title{
COMPORTAMENTO SUICIDA: 0 PARADOXO VIDA E MORTE EM MEIO À PANDEMIA DE COVID-19
}

Natália Gallo Mendes Ferracioli

Faculdade de Filosofia, Ciências e Letras de Ribeirão Preto Universidade de São Paulo

Wanderlei Abadio de Oliveira

Pontifícia Universidade Católica de Campinas

PUC-Campinas

Érika Arantes de Oliveira-Cardoso

Faculdade de Filosofia, Ciências e Letras de Ribeirão Preto

Universidade de São Paulo

\section{Clarissa Mendonça Corradi-Webster}

Faculdade de Filosofia, Ciências e Letras de Ribeirão Preto

Universidade de São Paulo

Eduardo Name Risk

Centro de Educação e Ciências Humanas

Universidade Federal de São Carlos

\section{Manoel Antônio dos Santos}

Faculdade de Filosofia, Ciências e Letras de Ribeirão Preto Universidade de São Paulo
Recebido em: 12/08/2020

$1^{\text {a }}$ revisão em: 16/06/2021

Aceito em: 05/07/2021 


\section{RESUMO}

A pandemia da COVID-19 provoca inúmeros impactos na saúde mental, que variam desde reações esperadas de estresse agudo até agravos que podem descompensar condições psicopatológicas preexistentes ou deflagrar novas. $\mathrm{O}$ objetivo deste estudo é identificar e analisar fatores de risco para o suicídio que podem ser exacerbados pela situação de emergência sanitária global. A revisão narrativa permitiu mapear os seguintes fatores de risco: luto complicado, insegurança econômica, isolamento social, violência doméstica, abuso de álcool e drogas, barreiras no acesso ao sistema de saúde e às redes de suporte comunitário, psicossocial e espiritual, riscos inerentes às situações de hospitalização e vulnerabilidade dos profissionais da linha de frente. Também foram abordados aspectos que podem funcionar como proteção e métodos de prevenção/posvenção ao suicídio. A situação pandêmica eleva a vulnerabilidade ao comportamento suicida, porém os efeitos deletérios podem ser mitigados com estratégias de proteção à saúde mental, que devem ter prioridade na agenda de saúde pública.

Palavras-chave: suicídio; comportamento suicida; prevenção do suicídio; pandemia; COVID-19.

\section{SUICIDE BEHAVIOR: LIFE AND DEATH PARADOX IN THE MIDST OF THE COVID-19 PANDEMIC}

\section{ABSTRACT}

The COVID-19 pandemic causes numerous impacts on the mental health, ranging from expected reactions of acute stress to more pronounced complications, which can decompensate pre-existing psychopathological conditions or trigger new ones. The purpose of this study is to identify and analyze risk factors for suicide that may be exacerbated by the global health emergency situation. The narrative review allowed mapping the following risk factors: complicated grief, economic insecurity, social isolation, domestic violence, alcohol and drug abuse, barriers in accessing the health system and community, psychosocial and spiritual support networks, risks inherent to hospitalization situations, and vulnerability of frontline professionals. Aspects that can act as protection and methods of suicide prevention and postvention were also addressed. The pandemic situation raises the vulnerability of people to suicidal behavior, but the deleterious effects can be mitigated with mental health protection strategies, which should have priority on the public health agenda.

Keywords: suicide; suicidal behavior; suicide prevention; pandemics; COVID-19. 


\section{LA CONDUCTA SUICIDA: LA PARADOJA DE LA VIDA Y LA MUERTE EN EL CONTEXTO DE LA PANDEMIA DE COVID-19}

\section{RESUMEN}

La pandemia de COVID-19 causa numerosos efectos en la salud mental de las personas, desde las esperadas reacciones de estrés agudo hasta complicaciones que pueden descompensar condiciones psiquiátricas preexistentes 0 desencadenar otras nuevas. El objetivo de este estudio es identificar y analizar los factores de riesgo de suicidio que pueden verse exacerbados por la situación de emergencia sanitaria mundial. La revisión narrativa permitió mapear los siguientes factores de riesgo: duelo complicado, inseguridad económica, aislamiento social, violencia doméstica, abuso de alcohol y drogas, barreras de acceso al sistema de salud y a las redes de apoyo comunitario, psicosocial y espiritual, riesgos inherentes a las situaciones de hospitalización y vulnerabilidad de los profesionales de primera línea. También se abordaron los factores que pueden funcionar como protección y los métodos de prevención y postvención del suicidio. La situación de pandemia aumenta la vulnerabilidad al comportamiento suicida, pero los efectos nocivos pueden mitigarse con estrategias de protección de la salud mental, a las que debería darse prioridad en la agenda de salud pública.

Palabras clave: suicidio; conducta suicida; prevención del suicidio; pandemia; COVID-19. 


\section{INTRODUÇÃO}

O conceito moderno de pandemia refere-se a uma epidemia de grandes proporções, que ultrapassa barreiras geográficas, disseminando-se por vários países e por mais de um continente (Rezende, 1998). A pandemia da COVID-19, doença causada pelo novo coronavírus (SARS-CoV-2), tem sido considerada como o maior desafio sanitário mundial em mais de um século (Werneck \& Carvalho, 2020). Até 28 de junho de 2021, com cerca de um ano e meio desde o registro do primeiro caso na China, o número de pessoas infectadas globalmente havia alcançado a impressionante marca de 180.492 .131 casos de COVID-19 (do quais 18.417.113 infectados foram registrados no Brasil) e 3.916.771 óbitos no mundo, dos quais 513.544 foram contabilizados em nosso país (World Health Organization [WHO], 2021). Deve-se levar em consideração, ainda, a possibilidade de que os dados oficiais estejam subestimados devido a diversas deficiências na forma como são contabilizados.

O combate ao novo coronavírus solicita medidas sanitárias que impõem severas restrições à circulação e interação social (Santos, Oliveira, \& Oliveira-Santos, 2020). Devido às peculiares características de disseminação do patógeno por via respiratória, ações foram implementadas a fim de reduzir o contato humano. Vattimo (2020) afirma que nossos valores fundamentais enquanto sociedade foram afetados com a necessidade de confinamento domiciliar e distanciamento social compulsório, o que coloca em xeque até mesmo o princípio da liberdade individual, um dos pilares das democracias modernas. Embora se reconheça a necessidade de adotar tais medidas não farmacológicas complementares à imunização em massa, visando a reduzir as taxas de novas infecções e, consequentemente, de letalidade, o potencial dessas ações para produzir efeitos adversos à saúde mental é apreciável (Ferreira, 2021; Scorsolini-Comin, Rossato, \& Santos, 2020).

Entre os impactos das medidas de combate à pandemia de COVID-19 sobre a saúde mental encontramos desde reações esperadas de estresse agudo, decorrentes da necessidade de ajustamentos e adaptações a uma rotina totalmente nova e até então impensável, até o agravamento de sintomas prévios e aumento dos níveis de sofrimento psíquico (Ministério da Saúde, 2020). Entre os possíveis agravos psíquicos encontram-se os mais diversos quadros psiquiátricos, em especial os transtornos de ansiedade (aguda ou generalizada, transtorno obsessivo-compulsivo, entre outros), transtornos de humor (depressão maior, transtorno bipolar), transtornos psicóticos, abuso de álcool e outras substâncias psicoativas, além da possibilidade de ocorrência de luto traumático ou complicado. Tempos de pandemia também podem favorecer a descompensação de condições psiquiátricas preexistentes, porém também é esperado o surgimento de novos casos relacionados às vivências de sobrecarga psíquica decorrente dessa situaçãolimite (Braga, Oliveira, \& Santos, 2020). A maior suscetibilidade aos sintomas e quadros psicopatológicos eleva o risco de comportamento suicida no período 
pandêmico, e a coexistência com vulnerabilidades anteriores pode potencializar esses agravos.

Dados epidemiológicos indicam que cerca de 800 mil pessoas se suicidam por ano em todo o mundo (WHO, 2020). Porém, deve-se considerar a possibilidade de que os registros estejam subestimados, uma vez que, provavelmente, parte substancial dos óbitos por autoextermínio não tem sido contabilizada como tal, dada a dificuldade de confirmação de intencionalidade e os próprios tabus e estigmas que envolvem a situação e o tema da morte por violência autoinfligida (Fukumitsu \& Kovács, 2015; Schmidt, Gabarra, \& Gonçalves, 2011). Ademais, quando se trata de tentativas, torna-se ainda mais difícil precisar o quantitativo. Estima-se que ocorram cerca de 20 vezes mais tentativas do que o suicídio consumado (Botega, 2014; Ferreira Junior, 2015), mas há dados que apontam que esse número pode ser até 40 vezes maior do que o registrado (Bertolote, Mello-Santos, \& Botega, 2010). Avalia-se que apenas um terço desses casos chega a ser atendido em prontosocorro ou unidades de emergência (Ministério da Saúde, 2020). É relevante destacar que uma tentativa de suicídio é considerada o principal fator de risco para sua consumação (Botega, 2015) e pode aumentar em até 100 vezes o risco de que o evento ocorra no futuro (Ministério da Saúde, 2020), de forma que a atenção deve ser redobrada nesses casos.

O suicídio é considerado um grave problema de saúde pública. No entanto, é um comportamento evitável mediante intervenções oportunas, respaldadas na literatura e baseadas em evidências científicas, muitas vezes de baixo custo operacional (WHO, 2020). Para que as respostas nacionais sejam eficazes, é necessária uma estratégia abrangente e multissetorial de prevenção (Santos, 2017). Sabe-se de longa data que o fenômeno do suicídio é multifatorial e multideterminado, e que em sua efetivação está presente a interação de fatores individuais e pressões externas (Cassorla, 2017; Pereira \& Cardoso, 2015). Embora essa inter-relação seja variável e multidimensional, é possível identificar as trajetórias que apontam na direção do risco. Tais fatores são divididos em predisponentes e precipitantes. Os primeiros dizem respeito a elementos constitutivos orgânicos, fatores socioculturais, traços de personalidade e história de vida do indivíduo, enquanto os demais são os fatores desencadeantes do ato, que geralmente estão ligados à possibilidade de acesso a meios letais e deflagrados por alguma situação de perda; essa perda pode ser real, imaginada ou mesmo temida (Botega, 2015).

As motivações do comportamento suicida são complexas e não podem ser reduzidas ao exame de fatores precipitantes ou atribuídas a uma causalidade única. O fenômeno suicida ocorre ao longo de um continuum, que perpassa a ideação, ameaças, gestos autolesivos e tentativas de autoagressão até o ato consumado de autoextermínio (Ferracioli et al., 2019; Vedana et al., 2017).

Estudo aponta que as chamadas mortes por desespero (relacionadas ao abuso de álcool, drogas e suicídio) devem ser vistas, no contexto da propagação 
descontrolada da infecção pelo novo coronavírus nos Estados Unidos, como uma possível epidemia dentro da pandemia (Petterson, Westfall, \& Miller, 2020). Os pesquisadores levaram em consideração três fatores principais: crise econômica aguda e aumento vertiginoso do desemprego, isolamento social compulsório por meses (e, possivelmente, residual por anos) e as incertezas crescentes em relação ao futuro. Foram desenhados nove cenários diferentes, desde o mais favorável (em que haveria uma recuperação rápida da economia e baixo impacto na taxa de desemprego) até o mais desfavorável (recuperação lenta e grande impacto da crise no desemprego), o que levou à estimativa de taxas de suicídio entre 27.644 e 154.037, a depender do cenário que se consolidar, com uma média prevista de 68.000 mortes por desespero apenas nos Estados Unidos. No Brasil, os socorristas do Serviço de Atendimento Móvel de Urgência (SAMU) da cidade de São Paulo, embora não disponham do amparo de estatísticas oficiais, estimam que houve aumento no atendimento a tentativas de suicídio e casos de suicídio consumado nos primeiros meses da quarentena (Sakamoto, 2020).

É fato que a pandemia não deve ser apontada como motivo isolado para a deflagração do comportamento suicida, considerando não ser factível atribuir qualquer tipo de unicausalidade para o fenômeno (Santos, 2017). Tendo em vista a magnitude e intensidade da crise global deflagrada pela COVID-19, com suas características peculiares e consequências adversas, torna-se relevante identificar e analisar os fatores de risco que devem chamar a atenção dos profissionais de saúde e educação, agentes públicos governamentais, formuladores de políticas públicas, comunidades e famílias, na situação de anormalidade instaurada pelo novo coronavírus.

Com o intuito de fornecer elementos que favoreçam a articulação do conhecimento científico sedimentado acerca do comportamento suicida e o que já se tem documentado em relação ao cenário pandêmico, o objetivo deste estudo é identificar e analisar fatores de risco para o suicídio que podem ser exacerbados pela situação de emergência sanitária global.

\section{MÉTODO}

Este estudo configura-se como uma reflexão teórica, fundamentada em revisão narrativa da literatura. Este método se mostra adequado para alcançar o objetivo proposto, uma vez que permite realizar busca e seleção mais ampla, flexível e inclusiva de publicações que contemplam a temática de interesse, a partir da consulta a diversas fontes e adoção de diferentes vértices de análise (Gil, 2002). Isso possibilita ao leitor, em um curto período de tempo, ter acesso à produção de conhecimento atualizado e a informações relevantes que favorecem a prática clínica (Rother, 2007). A finalidade deste tipo de estudo é fornecer subsídios para a identificação e análise dos fatores de risco para o suicídio que podem estar exacerbados durante a travessia da pandemia da COVID-19, de modo que ações de proteção à vida e políticas públicas possam ser planejadas e implementadas, incluindo as necessidades específicas de saúde mental. 
O material selecionado nesta investigação contempla um amplo leque de fontes de disseminação do conhecimento científico, como artigos recuperados de bases indexadoras nacionais e internacionais, a saber: SciELO, PubMed, PePSIC e Google Acadêmico, bem como livros, cartilhas, relatórios, diretrizes sobre comportamento suicida, além de textos e notícias acerca da pandemia veiculadas na mídia. Tais produções foram integradas e balizadas com a experiência clínica psicológica dos pesquisadores/as, responsáveis pela seleção do material relevante para elucidação do tema proposto.

\section{RESULTADOS E DISCUSSÃO}

A partir dos estudos sobre suicídio identificados no material selecionado para compor a presente revisão, foi possível delinear a complexa relação entre os principais fatores de risco para o comportamento suicida e a situação de crise sanitária global deflagrada pela pandemia da COVID-19, bem como descrever e refletir sobre possíveis fatores de proteção.

\section{OS FATORES DE RISCO}

Já são bem documentados na literatura os diversos fatores de risco para o comportamento suicida (Bertolote, Mello-Santos \& Botega, 2010; Botega, 2015; Cassorla, 2017; Ferracioli et al., 2019; Ferreira Junior, 2015; Joiner, 2007). Em conformidade com os objetivos traçados por este estudo, interessa-nos identificar aqueles fatores que têm maior potencial de exacerbação no cenário pandêmico.

\section{QUEBRA DA ILUSÃO DO MUNDO PRESUMIDO E LUTO COMPLICADO NA PANDEMIA: UMA CRISE DENTRO DA CRISE}

Com a pandemia, vivemos uma situação de crise, definida como um evento circunstancial externo e extraordinário que foge ao controle do indivíduo e que exige uma resposta de ajustamento psicossocial. O evento ameaçador deflagra um estado psicológico também de crise, favorecendo vivências peculiares. Botega (2015) aponta que a crise pode levar ao colapso existencial caso ultrapasse a capacidade pessoal de reação e adaptação, com vivências de falta de perspectiva de solução, sentimento de incapacidade e esgotamento de recursos, o que, em alguns indivíduos mais suscetíveis, pode elevar a vulnerabilidade ao suicídio, que algumas vezes passa a ser encarado como única solução para um momento insuportável.

O psiquiatra britânico Colin Murray Parkes (1998) desenvolveu o conceito de "mundo presumido" para referir-se ao modelo de mundo que cada indivíduo traz consigo, que é formado por vários componentes, obviamente diferentes para cada um, dada a singularidade da subjetividade, mas que no geral abarcam nossa concepção sobre nós mesmos, nossa família, nossas habilidades e competências, 
e o significado que atribuímos às experiências individuais. Basicamente, nosso mundo presumido é tudo aquilo que consideramos garantido em nossas vidas. Uma situação aguda como a despertada pela pandemia representa um abalo sísmico nas nossas concepções cristalizadas e, por conseguinte, uma quebra de nosso mundo presumido. Na medida em que a situação de exceção se prolonga indefinidamente, novos ajustes são necessários para lidar com os estímulos perturbadores, o que vai exigir um esforço reiterado e um trabalho de luto para reorganizar e ressignificar as vivências e perdas decorrentes, reconstruindo internamente uma nova forma de estar no (novo) mundo. Nesse processo de ajustamento, é esperado que surjam incertezas quanto ao futuro, que podem acentuar sentimentos de desamparo e desespero caso venham acompanhados de imprevisilidade e desesperança, podendo criar uma "tempestade perfeita" para desfechos dramáticos (Grupe \& Nitschke, 2013). Se nenhuma medida interventiva for tomada, configura-se um cenário altamente conturbado, que pode funcionar como gatilho para deflagrar condutas sintomáticas que visem à cessação do sofrimento insuportável. Estão criadas, assim, as condições para aumento do risco de comportamento suicida.

Conforme mencionado anteriormente, os fatores precipitantes muitas vezes estão associados a algum tipo de perda, adversidade, crises atuais ou recentes. Por seu caráter praticamente universal no momento de pandemia, faz-se necessário aprofundar a discussão acerca deste aspecto, já que inevitavelmente sofremos algum tipo de perda em consequência da crise instaurada. Na verdade, durante a pandemia enfrentamos diversas perdas concomitantes e nossas famílias foram impactadas de maneira singular. O primeiro prejuízo de que nos demos conta foi relacionado à organização da rotina e dos afazeres diários: compromissos, eventos, reuniões, encontros e comemorações foram adiados ou cancelados. Planos foram cancelados e sonhos postergados. Até mesmo a presença das crianças e adolescentes na escola teve de ser interrompida, as aulas presenciais foram suspensas e o ensino teve de ser remodelado para se adaptar ao ambiente virtual (Oliveira, W.A. et al., 2020c). Perdemos a presença, o contato físico e o toque de pessoas externas ao nosso núcleo familiar: um simples aperto de mão passou a representar risco de vida. O próprio autocuidado ficou prejudicado ao perdermos a possibilidade de frequentarmos academias, quadras, parques e locais apropriados para a prática de atividades físicas, e também ao temermos comparecer às instituições de saúde para dar continuidade a tratamentos que não configurassem urgência.

Perdemos as possibilidades de lazer e distração tais como as conhecíamos (museus, cinemas, teatros, restaurantes, praias, espaços públicos). Finais de semana e feriados ficaram dissolvidos em meio à nova rotina dentro de casa. Perdemos a tranquilidade e até mesmo o direito de ir e vir. Deixamos, no mínimo, de usufruir da vida tal como a conhecíamos. Em muitos casos, a convivência próxima elevou a temperatura dos conflitos familiares, acentuando a possibilidade de rupturas nos relacionamentos e de exacerbação da violência interpessoal. Também sofremos com a interrupção prematura de projetos e perdas financeiras, e muitas pessoas 
perderam seus empregos e até mesmo sua moradia devido à inadimplência. Acima de tudo, inúmeras (e insubstituíveis) vidas foram precocemente perdidas: desde pessoas distantes que contraíram e morreram em decorrência de complicações da COVID-19, até pessoas próximas de nosso círculo social e familiar. Muitos adotaram uma postura negacionista diante da gravidade da pandemia, até que a tragédia começasse a chegar nas casas de pessoas próximas, amigos e familiares, e as estatísticas oficiais deixaram de retratar números frios e passaram a ter nomes e rostos. As dores suscitadas pelas perdas de pessoas significativas ficaram escancaradas e as feridas abertas ainda estão longe de cicatrizarem.

Além das consequências mais imediatas ligadas às situações de perdas, não podemos deixar de considerar os riscos a médio e longo prazo que podem advir da sobreposição de lutos acumulados e não elaborados. Os rituais de despedida foram abreviados, os ritos funerários foram suprimidos ou totalmente modificados, com restrição de acesso de visitas, diminuição na duração ou impedimento de velórios presenciais e realização de serviços funerários virtuais (Oliveira-Cardoso et al., 2020). Essas condições adversas abrem caminho para a possibilidade de ocorrência de luto traumático e, portanto, aumentam o risco de luto complicado (Parkes, 1998; Oliveira-Cardoso et al., 2020; Siqueira et al., 2019), que traz riscos à saúde mental, elevando a vulnerabilidade pessoal ao suicídio.

O impacto relativo das perdas depende de vários fatores pessoais e sociais, sendo também proporcional à idade. Barbosa, Melchiori e Neme (2011) constataram que a morte é abordada com maior inquietação por adolescentes e adultos, visto que eles ainda têm projetos futuros. Já os idosos encaram a finitude com maior serenidade, como parte do processo natural da vida, temendo por outro lado adoecerem e se tornarem dependentes ou morrerem com sofrimento.

\section{EM MEIO A TANTAS PERDAS, O AGRAVAMENTO DA INSEGURANÇA ECONÔMICA}

Entre as situações de perdas que potencialmente elevam o risco de suicídio, destaca-se nos estudos o impacto pessoal e familiar do agravamento da crise econômica (Brown \& Schuman, 2020; Gunnell et al., 2020; Ministério da Saúde, 2020; Petterson et al., 2020; Reger, Stanley, \& Joiner, 2020). A escalada exponencial de mortes em decorrência de complicações da COVID-19 e a instabilidade da atividade econômica provocadas pela pandemia ampliaram o cenário de incertezas. O estado de calamidade sanitária agravou a insegurança econômica e alimentar das famílias vulneráveis e a condução errática da crise no Brasil dificultou ainda mais a retomada da economia, instaurando uma "tempestade perfeita" que alimenta um ciclo vicioso. Com o fechamento repentino do comércio e das escolas, cancelamento de eventos e instabilidade nos mercados, instalou-se um cenário de recessão e desemprego maciço, fatores tradicionalmente associados a taxas mais elevadas de suicídio quando comparados aos períodos de prosperidade econômica (Reger et al., 2020). Estudos anteriores à pandemia corroboram essa tendência e evidenciam que, em períodos de desemprego e recessão, incremento de um ponto percentual nestas taxas eleva entre 1,3\% e 1,6\% a taxa de suicídios, e 
em 3,3\% o consumo de substâncias psicoativas (Petterson et al., 2020). O uso abusivo de substâncias é outro fator de risco sobre o qual discorreremos mais adiante.

\section{DISTANCIAMENTO FÍSICO É O MESMO QUE ISOLAMENTO SOCIAL?}

Uma das decorrências da atual experiência coletiva de crise está ligada à necessidade de manter o confinamento doméstico (que tem sido popularizada com o termo "quarentena"), uma das medidas de distanciamento social, complementar a outras estratégias não farmacológicas preconizadas para evitar a infecção pelo novo coronavírus, como lavar regularmente as mãos e usar a máscara de proteção facial. Permanecer em casa o máximo de tempo possível, evitando de se expor ao contato próximo com outras pessoas, impõem a manutenção por tempo prolongado do afastamento do convívio social, uma atitude conhecida como um dos mais relevantes fatores predisponentes ao comportamento suicida (Botega, 2015; Ferracioli et al., 2019; Ferreira Junior, 2015; Joiner, 2007). É claro que manter o distanciamento físico não significa necessariamente isolamento social. Porém, sabemos que a solidão e a fragilidade ou ausência de contatos e vínculos afetivos significativos diminuem as possibilidades de que alguém atente ao sofrimento do outro, o que permitiria mantê-lo sob vigilância, com disponibilidade para fornecer suporte psicossocial e auxiliar no encaminhamento e acompanhamento aos profissionais competentes quando necessário. Do ponto de vista do comportamento suicida, a restrição de contato social é a estratégia mais preocupante, adotada para a contenção da disseminação da COVID-19, sobretudo quando se estende por período prolongado (Reger et al., 2020).

Além disso, levando-se em conta que o principal local onde as pessoas consumam o suicídio no Brasil é a própria casa, com 51\% das ocorrências (Botega, 2015; Ministério da Saúde, 2020), o confinamento domiciliar, com ausência de circulação de pessoas pelo local, nos casos em que o indivíduo vive sozinho, pode elevar exponencialmente esse risco. Atenção especial deve ser dedicada aos idosos, faixa etária cujos índices de suicídio têm aumentado nos últimos anos no contexto brasileiro (Ministério da Saúde, 2020). A falta de contato próximo com familiares e com a rede de apoio psicossocial torna os mais velhos particularmente sensíveis à solidão e isolamento. Com uma particularidade: na dinâmica da COVID-19, os idosos temem contrair a forma mais grave da doença e se tornar um fardo para os familiares, conforme demonstrou um estudo realizado em Hong Kong sobre o impacto da epidemia de outras doenças respiratórias, como a Síndrome Respiratória Aguda Grave (SARS) (Ministério da Saúde, 2020). Nesse contexto, é preciso fazer uma distinção entre isolamento social e distanciamento físico: apenas este último deve ocorrer para prevenção da disseminação e contágio pelo novo coronavírus. Na atualidade, há inúmeros recursos tecnológicos de interação online, inclusive com acesso a métodos de cuidado pessoal para que o contato interpessoal seja preservado, a despeito da necessidade de manter o isolamento físico. Vale apontar, porém, que principalmente no caso de crianças e adolescentes é necessário manter atenção à forma como as mídias eletrônicas são utilizadas, 
bem como os conteúdos que estão sendo visualizados, uma vez que existem grupos que fazem apologia do suicídio, como o sinistro jogo da "Baleia Azul". São páginas de fácil acesso no ambiente on-line que incitam a violência, e glorificam e normalizam o comportamento autolesivo (Ministério da Saúde, 2020).

\section{VIOLÊNCIA DOMÉSTICA}

Outro agravante da situação de confinamento domiciliar é a maior exposição de mulheres, crianças e adolescentes à violência doméstica (Oliveira, W.A. et al., 2020b; Soares et al., 2020). Considerando-se que, na maioria dos casos, esse tipo de violência é perpetrado pelos próprios familiares, em contextos nos quais já ocorria anteriormente, a intensa convivência familiar pode exacerbar conflitos, somada ao acúmulo de tensões e sobrecargas de diversas ordens (aumento de responsabilidades e afazeres domésticos, trabalho em casa dos pais mesclado com o ensino remoto dos filhos, desemprego, perda de renda e falta de apoio governamental). Problemas relacionais, com presença de manifestações de violência entre parceiros íntimos, estão associados a maiores taxas de homicídio seguido de suicídio - o que deve ser uma preocupação adicional neste contexto, no qual se observa aumento do feminicídio (Brown \& Schuman, 2020).

\section{CONFINAMENTO DOMICILIAR E USO DE SUBSTÂNCIAS: MISTURA EXPLOSIVA OU APOSTA ARRISCADA?}

Outra situação que tem se mostrado comum durante o período de confinamento domiciliar prolongado é a elevação dos níveis de consumo de álcool e de outras substâncias psicoativas. Na tentativa de conter angústias, ansiedade e sofrimento em geral, muitas pessoas recorrem a esses comportamentos de abuso, que por si só já representam um risco potencial, uma vez que a busca do equilíbrio pode ficar ainda mais prejudicada diante do cenário adverso. Brown e Schuman (2020) apontam que a disponibilidade de álcool, inclusive com serviços de entrega domiciliar, na ausência de uma capacidade de ter acesso prontamente a alternativas saudáveis de redução de estresse, como academias de ginástica e congregações religiosas, pode contribuir para elevar o uso indevido de sustâncias durante a pandemia. O uso nocivo de bebidas alcoólicas e outras substâncias eleva o risco de suicídio, uma vez que o abuso aumenta o potencial de violência e impulsividade dos comportamentos, prejudica a crítica e intensifica o grau de letalidade do método utilizado para a tentativa de suicídio (Botega, 2015; Brown \& Schuman, 2020; Gunnell et al., 2020; Reger et al., 2020). Pelo menos um quarto das pessoas que se suicidam apresentavam transtornos decorrentes do abuso de álcool e em cerca de um terço à metade das vítimas de suicídio necropsiadas foi detectada a presença de álcool no sangue (Botega, 2015).

O abuso de álcool e de outras substâncias tem íntima relação com outros fatores de risco para o suicídio, constituindo uma via de mão dupla: ao mesmo tempo que esse comportamento pode ser desencadeado e agravado por condições psíquicas e sociais desfavoráveis, também repercute nas várias esferas da vida do indivíduo, retroalimentando-se em um ciclo pernicioso e perigoso. É comum que usuários 
crônicos apresentem comprometimentos em diversas esferas, como na saúde física, outros transtornos psiquiátricos associados (comorbidades como depressão maior, transtorno afetivo bipolar, ansiedade, transtornos de personalidade), vida pessoal e relacionamentos conturbados, laços sociais fragilizados, vida profissional marcada por perdas e frustrações. Além disso, essas pessoas podem desenvolver abstinência e apresentar, em decorrência, quadros de confusão mental, sintomas psicóticos e impulsividade descontrolada (Botega, 2015). Se essa combinação de fatores de risco já tem forte potencial explosivo em uma situação de mundo corriqueira, quando somada às condições de desarranjo de vida precipitado pela pandemia torna o cenário ainda mais caótico e com consequências imprevisíveis. Conforme apontam Brown e Schuman (2020), esse contexto constitui uma "tempestade perfeita" (perfect storm) de condições que predispõem ao suicídio.

\section{BARREIRAS NO ACESSO AO SISTEMA DE SAÚDE: UM PARADOXO NO CUIDADO}

Outro risco associado à pandemia é que as medidas que restringem ao máximo o contato social têm potencial de prejudicar outros cuidados em saúde, além de abalar a organização da assistência. Por medo de se expor e contrair o vírus, muitas pessoas deixam de realizar seus tratamentos habituais, inclusive cuidados com a saúde mental, diminuindo a adesão ao seguimento terapêutico e medicamentoso, o que contribui para exacerbar seus sintomas e agravar o quadro clínico. Doenças orgânicas, por si, elevam o risco de suicídio para alguns indivíduos, especialmente os mais idosos (Botega, 2015; Reger et al., 2020). O agravamento dos sintomas pode intensificar a sensação de perda da autoeficácia, o sentimento de poder exercer algum controle sobre os principais acontecimentos de nossas vidas, intensificando a desesperança (Arruda-Colli, Sansom-Daly, Santos, \& Wiener, 2018). Mas não apenas os temores individuais podem levar à interrupção dos cuidados recomendados em termos de saúde mental. O acesso aos serviços é drasticamente reduzido ou interrompido em muitos contextos: em várias localidades, as instalações originalmente destinadas a essa finalidade foram adaptadas e convertidas em setores para atendimento exclusivo a pessoas com COVID-19. No contexto brasileiro, trabalhadores de saúde foram transferidos em regime de urgência para suprir as lacunas de profissionais especializados na linha de frente, muitos dos quais afastados por terem sido infectados ou estarem esgotados (Teixeira et al., 2020). Além disso, os autores explicitam que os recursos e insumos médicos se tornaram escassos e disputados no mercado mundial, e outros problemas de saúde passaram para segundo plano na visão dos gestores, por não serem considerados prioridade no momento de emergência e iminência de colapso do sistema de saúde.

Inúmeros sistemas presenciais de assistência à saúde mental foram interrompidos para conter a ameaça de disseminação do vírus e também em consequência das baixas por contaminação maciça de profissionais das equipes de saúde mental. Voluntários de diferentes serviços comunitários de suporte, como organizações e grupos de mútua ajuda para dependentes de álcool e drogas, ficaram 
impossibilitados de se reunirem. Os serviços de emergência médica ficaram sobrecarregados e os leitos hospitalares superlotados, afetando negativamente o atendimento de sobreviventes de tentativas de suicídio, dificultando o tratamento, bem como o seguimento que é crucial na posvenção (Reger et al., 2020).

\section{REDES DE SUPORTE COMUNITÁRIO, PSICOSSOCIAL E ESPIRITUAL: RELIGIOSIDADE E ESPIRITUALIDADE}

O convívio com cifras superlativas de mortes, reiteradas diariamente durante a pandemia, instaurou um cenário insólito de desamparo social. A morte nunca esteve tão presente no cotidiano das famílias. Nesse contexto, a religiosidade pode ser um fator de proteção ao suicídio por uma dupla via: pelo significado pessoal de aderir a um sistema de crenças sobre a vida, morte e eventual vida após a morte (Benites, Neme, \& Santos, 2017; Borges et al., 2006), e pelo estabelecimento de uma rede de apoio social constituída pelos adeptos da religião de escolha (Botega, 2015; Freitas, Oliveira-Cardoso, \& Santos, 2017). As restrições impostas pelo momento de pandemia podem abalar ambas as dimensões, uma vez que muitas crenças religiosas podem ser questionadas caso predominem sentimentos de insegurança e desesperança. Ademais, o acesso a cerimônias, cultos, celebrações, e o apoio direto proporcionado por membros da religião ficam reduzidos durante a quarentena.

Deve-se levar em consideração que muitas pessoas não têm meios de acesso a uma conexão de internet de qualidade ou mesmo destreza suficiente para manejar a tecnologia de comunicação a distância para participarem de cultos remotos. Mesmo quando existe a oferta desses recursos por parte das congregações e adesão individual a essa modalidade de participação remota nos serviços religiosos, o adepto não estará mais acompanhado por seus pares de fé religiosa como anteriormente, quando podia compartilhar da proximidade física. O sentimento de pertencimento, renovado nos encontros e celebrações religiosas, favorece a interação e fortalece o intercâmbio de experiências e calor afetivo, potencializando os benefícios do apoio mútuo, especialmente em um período de crise sanitária no qual as pessoas estão expostas cotidianamente à dor do desamparo e da terminalidade (Cardoso, Mastropietro, Santos, \& Voltarelli, 2010).

\section{RISCOS INERENTES ÀS SITUAÇÕES DE HOSPITALIZAÇÃO E SUICÍDIO}

Pacientes internados em hospital geral, independentemente da situação de pandemia, têm de três a cinco vezes mais risco de suicídio do que a população em geral (Botega, 2015; Ministério da Saúde, 2020). Diversas condições combinam-se neste tipo de situação, como por exemplo as vivências psicossociais de sofrimento, desespero e desesperança ligadas ao diagnóstico (ou a falta dele), prognóstico e exigências do tratamento, além do acesso fácil a métodos letais (objetos perfurocortantes, medicamentos, janelas sem grades ou telas) e a possibilidade de ocorrência de delirium, com agitação psicomotora e impulsividade acentuada. Entre os locais de maior ocorrência de suicídios no Brasil, as instituições 
hospitalares figuram em segundo lugar (26\%), atrás apenas das residências (Botega, 2015).

No contexto da COVID-19, podem ocorrer reações agudas ao diagnóstico, ou à espera pelo resultado dos exames laboratoriais, lembrando que os kits diagnósticos não estão disponíveis para toda a população e que os testes podem apresentar resultados não confiáveis, e até mesmo o receio na discussão sobre o prognóstico, com incertezas e temores antecipados de sofrimento inerentes às próprias manifestações da doença, de forma que pacientes com histórico de tentativas de suicídio devem receber atenção redobrada (Botega, 2014). Além disso, indivíduos hospitalizados precisam ficar isolados de seus familiares e amigos, o que eleva o sofrimento psíquico para todos os envolvidos, inclusive para os familiares. Nesse contexto de adversidades somadas, devem ser favorecidas estratégias alternativas de interação social, como por exemplo a realização de videochamadas ou a troca de áudios, ou devem ser testadas outras possibilidades de comunicação remota para atenuar o sentimento de isolamento e solidão.

\section{OS RISCOS DE QUEM ESTÁ NA TRINCHEIRA: VULNERABILIDADE DOS PROFISSIONAIS DE SAÚDE NA LINHA DE FRENTE}

Em contextos de normalidade, médicos apresentam taxa de suicídio cinco vezes maior do que a população em geral, e entre enfermeiros e assistentes sociais este risco também é elevado (Botega, 2015). Algumas hipóteses são formuladas na tentativa de buscar explicações plausíveis para esse fenômeno, como o acesso facilitado a métodos letais e conhecimento acerca de como utilizá-los, tendência para a agregação de indivíduos com transtornos psiquiátricos em algumas categorias profissionais e estressores específicos envolvidos no exercício dessas profissões. No cenário pandêmico, tais estressores podem ficar absolutamente exacerbados. Os impactos sobre o equilíbrio mental dos trabalhadores da saúde também são influenciados pelas atitudes frente à morte e pelos significados construídos pelos profissionais acerca da finitude (Santos M.A. \& Hormanez, 2013; Santos M.A., Aoki, \& Oliveira-Cardoso, 2013).

Ainda não foi dada atenção suficiente aos graves riscos à saúde mental dos profissionais da chamada linha de frente do combate à COVID-19. As preocupações são excessivamente centradas nos riscos à integridade física, negligenciando-se as questões de saúde mental, o que é justificável em países que ainda não tratam seus profissionais de saúde com a dignidade que eles merecem. A indisponibilidade de equipamentos de proteção individual (EPIs) adequados em vários serviços, o risco elevado de contraírem o vírus e infectarem outras pessoas, inclusive familiares e entes queridos, as extenuantes cargas e escalas desumanas de trabalho, além da necessidade sem precedentes de lidar com dilemas bioéticos e decisões de vida ou morte, entre outros agravantes, tornam esses trabalhadores particularmente vulneráveis ao desenvolvimento de sintomas depressivos, ansiosos e de transtorno do estresse pós-traumático (Oliveira W.A., OliveiraCardoso, Silva, \& Santos, 2020a; WHO, 2021). 
Pensando em modos de atenuar essa vulnerabilidade, psicólogos, psiquiatrias, organizações, entidades e instituições criaram projetos disponibilizando sites e linhas telefônicas de suporte gratuito aos profissionais que estão atuando na pandemia, o que funciona como rede de proteção psicossocial, embora isoladamente possa não ser suficiente face aos desafios que se avolumam em um cenário de descontrole da COVID-19. Várias destas iniciativas estão listadas no site Mapa da Saúde Mental (2021), que divulga serviços de atendimento voluntário online e presencial de suporte psicológico aos trabalhadores de saúde durante a pandemia.

\section{OS FATORES DE PROTEÇÃO}

Embora muitos dos conhecidos fatores de risco para o comportamento suicida possam ser exacerbados com a pandemia e suas consequências, é importante observar que fatores de proteção também podem ser identificados, beneficiando alguns grupos sociais no processo de enfrentamento dos desafios emergentes no cenário de exceção que se instaurou com a COVID-19. Conhecer e dar visibilidade aos aspectos de proteção é uma estratégia crucial para vislumbrar possíveis vias de superação dos danos gerados pela pandemia.

\section{PRESENÇA FÍSICA A FAVOR DA DISPONIBILIDADE E ATENÇÃO EMOCIONAL}

Apesar dos já mencionados riscos de efeitos adversos decorrentes do confinamento domiciliar e das rupturas do cotidiano, que impuseram restrições e mudanças na rotina, é fato que muitos indivíduos e suas famílias evitaram se aproximar, sobretudo fisicamente. Psicologicamente, contudo, houve uma proximidade maior. Devido ao maior tempo de convívio familiar intensivo, as pessoas puderam estar mais próximas também do ponto de vista emocional. Em muitas famílias, isso contribuiu para que atentassem para as necessidades dos demais membros, o que pode funcionar como fator protetivo para algumas pessoas que, anteriormente, devido às ausências físicas e atenção voltada para as demandas do mundo externo, não estavam tão atentas ao comportamento e à dinâmica emocional dos familiares, especialmente pais que passam a maior parte do tempo fora de casa em relação aos filhos adolescentes e jovens (Bisby, 2020; Weissbourd, Batanova, Mclntyre, \& Torres, 2020).

A proximidade dos familiares pode inibir o comportamento suicida, uma vez que a pessoa não permanece sozinha em casa para conseguir planejar e executar o ato sem que outros membros percebam. Evidentemente, deve-se considerar o efeito oposto, no sentido de que o convívio diário forçado pode intensificar conflitos latentes, tensionando ao máximo as relações, incrementando a violência doméstica e funcionando como gatilho para a conduta suicida em alguns casos. De todo modo, é importante lembrar que prevenir o suicídio não é necessariamente sinônimo de cuidar (Botega, 2015) e impedir com que a pessoa se mate é apenas o primeiro passo para investir na atenção à saúde mental da pessoa, que deve ser referida a uma rede de apoio que inclua profissionais de diversas áreas e que agregue a família e outros sistemas de suporte social. Além disso, a eventual 
proteção oferecida pela maior proximidade dos familiares tem um caráter mais imediato, uma vez que, retomadas as atividades habituais no cenário póspandemia, seus efeitos de vigilância serão suprimidos.

\section{PULLING TOGETHER EFFECT: JUNTOS CONTRA UM INIMIGO EM COMUM}

Outro importante fator de proteção a ser registrado é o chamado pulling together effect (Ministério da Saúde, 2020; Reger et al., 2020). Trata-se de um efeito observado historicamente nos Estados Unidos, após alguns desastres e guerras. Geralmente, são mencionadas a Segunda Guerra Mundial, a Guerra FrancoPrussiana e a Guerra Civil Americana, como grandes catástrofes humanitárias nas quais as taxas de suicídio decaíram durante e logo após a cessação desses eventos (Joiner, 2007). O autor pontua que, em tempos de aguda crise nacional, as conexões sociais tendem a aumentar, as pessoas se unem e o sentimento de coesão e pertencimento se fortalece, apesar dos efeitos negativos da crise; isso pode ocorrer pelo fato de a atenção das pessoas estar direcionada a preocupações maiores do que os sofrimentos individuais gerados pela desorganização e caos social. Ao enfrentarem uma experiência difícil de modo compartilhado, muitas pessoas tendem a oferecer suporte recíproco, estreitando o contato social (Reger et al., 2020). Tais autores apontam também que, na situação de epidemias e pandemias, a percepção de algumas pessoas sobre saúde e mortalidade pode sofrer modificações. À medida que a morte passa a ser mais temida, a vida tende a ser revalorizada e passa a ser vista como o bem mais precioso, tornando o suicídio menos provável.

No entanto, é relatado que, no caso das guerras, o pulling together effect tende a se extinguir após o término do conflito, sendo que a fase subsequente é a desilusão, na qual as pessoas se sentem desencorajadas e abandonadas e podem cair prostradas em um estado de profundo desamparo, com suas forças exauridas e sob estresse crônico, o que pode perdurar por meses ou até anos (Brown \& Schuman, 2020). Assim, não devemos confiar demasiadamente nos efeitos duradouros desse fenômeno, justamente porque é preciso pensar que a proteção conferida talvez seja apenas em caráter imediato, de forma que, suprimidos seus benefícios quando a crise mais aguda passar, podemos ver ainda mais exacerbados os fatores agravantes, potencializando os riscos.

Além disso, no atual contexto brasileiro, em que se escancara uma política federal negacionista, instalam-se cisões que criam severas rupturas entre diferentes grupos na sociedade, dificultando e reduzindo o sentimento de compartilhamento, solidariedade e suporte mútuo. De fato, estudos corroboram que o impacto deste fator de proteção é bastante sensível a questões culturais, sociais e governamentais, apresentando relevância limitada em contextos individualistas que supervalorizam a independência e interpretam vulnerabilidades pessoais e pedidos de ajuda como sinais de fraqueza pessoal (Botega et al., 2006; Prado \& Freitas, 2020). 


\section{COMPREENDENDO AS ESFERAS DE PREVENÇÃO E SUA INTERAÇÃO COM A PANDEMIA}

É provável que os riscos de comportamento suicida sejam uma das maiores preocupações à medida que a pandemia se dissemina e produz efeitos de longo prazo na população, afetando os mais variados âmbitos, da economia à educação, especialmente entre grupos mais vulnerabilizados, de modo que a prevenção ao comportamento suicida deve assumir caráter urgente e permanente (Gunnel et al., 2020). Tais autores sugerem que, para maior efetividade, as intervenções devem ser baseadas na avaliação do grau de risco, conforme descreveremos a seguir.

\section{INTERVENÇÕES UNIVERSAIS}

As intervenções universais têm como alvo toda a população, sem distinguir indivíduos vulneráveis. Foca-se em alguns fatores de risco específicos, com o objetivo de melhorar a saúde mental e prevenir o suicídio. Nesse contexto, são necessárias ações coordenadas que envolvem diversos atores: governo (ações empreendidas em resposta a estressores financeiros, violência doméstica, consumo abusivo de álcool, acesso a serviços de saúde mental, controle de acesso a métodos letais); comunidades, pessoas, famílias e provedores de serviços de saúde mental (ações em resposta ao isolamento, solidão e luto) e mídia (responsabilidade ética na divulgação de notícias, baseada nos manuais de orientação e auto-regulação do setor, incluindo sempre informações sobre acesso a fontes e canais de ajuda).

\section{INTERVENÇÕES SELETIVAS E INDICADAS}

As intervenções seletivas e indicadas focalizam populações com risco aumentado e miram indivíduos com histórico de comportamento suicida. As ações são desenhadas para reduzir os riscos de agravamento dos fatores precipitantes. Envolvem também instâncias como o governo, profissionais e serviços de saúde mental e outros recursos comunitários, para assegurar a oferta de cuidados de diferentes tipos, como os que envolvem meios digitais de telemedicina e telepsicologia, além de linhas de apoio psicológico específicas para profissionais de saúde, oferecidas por pessoal com treinamento adequado.

Outra questão importante é como operacionalizar a coleta de dados acerca de ideação, tentativas e suicídios consumados, por meio de questionários, informações fornecidas por membros de linhas de ajuda, profissionais e serviços de saúde mental, bem como unidades de pronto-atendimento, uma vez que o conhecimento desses agentes poderá favorecer respostas oportunas a serem incorporadas nas políticas públicas (Gunnel et al., 2020). Os autores apontam, inclusive, que os impactos da pandemia sobre o comportamento suicida podem variar conforme as medidas públicas de saúde adotadas pelos diferentes países, bem como as estruturas sociais, culturais, demográficas e serviços de suporte existentes, incluindo disponibilidade de acesso a alternativas de atenção à saúde mental em plataformas digitais, em detrimento dos atendimentos presenciais. São 
esperados desfechos piores em contextos menos favorecidos, nos quais a adversidade econômica limita o acesso a recursos e o suporte adequado ao bemestar das pessoas.

Como ressaltado previamente, têm se mostrado promissoras as modificações e adaptações na assistência à saúde mental e na rede de suporte psicossocial, como a realização de consultas mediadas por tecnologia e as visitas domiciliares nos casos mais graves (Gunnell et al., 2020). Além disso, há evidências de que algumas intervenções de prevenção pela via remota contribuem para reduzir as taxas de suicídio, como breves contatos telefônicos e cartas ou e-mails que demonstrem interesse e cuidado em saúde. Essas estratégias podem ser particularmente significativas para os indivíduos que contraíram COVID-19 e apresentam fatores de risco para o suicídio (Reger et al., 2020).

\section{POSVENÇÃO}

A morte por suicídio é sempre violenta e chocante, além de ser percebida em nossa cultura como injustificável e inaceitável (Botega, 2015). O suicídio tem efeito sistêmico na vida de inúmeros integrantes da rede pessoal daquele que decidiu abreviar sua vida. Familiares, amigos, escola, trabalho, grupos religiosos e demais grupos sociais aos quais a pessoa pertencia são profundamente afetados pela ocorrência traumática. O choque desencadeado pela notícia é comumente acompanhado por sentimentos de autoculpabilização e responsabilização pelo falecimento, devido aos laços ambivalentes que as pessoas nutrem pelo suicida. Subjacente à consternação pode se ocultar a raiva latente pela pessoa que resolveu deixar a vida de forma voluntária e que em um determinado dia saiu de cena "sem pedir licença" para quem quer que seja. Isso não impede que expressões afetivas autênticas, tais como tristeza e saudade, sejam vivenciadas (Fukumitsu \& Kovács, 2015; Parkes, 1998).

Essas vivências emocionais intensas e paradoxais têm elevado potencial de desencadear o luto complicado (Parkes, 1998). O luto traumático, por sua vez, constitui fator de risco para que outros suicídios venham a ocorrer dentre as pessoas mais afetadas, os chamados sobreviventes enlutados por suicídio. Por essa razão o trabalho de posvenção é fundamental. Consiste em medidas e atividades de cuidado em saúde direcionadas às pessoas mais vulnerabilizadas com a perda súbita e traumática, com o intuito de auxiliar a ressignificação do luto e oferecer amparo para suas necessidades psicossociais (Fukumitsu \& Kovács, 2015). A posvenção pode ser realizada em perspectiva clínica ou de saúde pública, e envolve a oferta ativa de informação, suporte de grupos, assistência prática e psicoterapia (Ministério da Saúde, 2020) em uma perspectiva clínica que valoriza a atitude de acolhimento (Alexandre, Vasconcelos, Santos, \& Monteiro, 2019). No cenário da pandemia, tais ações se tornam ainda mais relevantes e devem ser viabilizadas por meio dos recursos disponíveis de interação, atendimento e ações de suporte, uma vez que a intensidade emocional da vivência e a sobreposição de lutos têm potencial desorganizador sobre a saúde mental. A implementação de programas 
de posvenção requer práticas interdisciplinares e intersetoriais que promovam autonomia e cidadania, a exemplo do que ocorre nos centros de atenção psicossocial, contribuindo assim para uma atenção baseada no território (Oliveira T.T.S.S., Fabrici, \& Santos, 2018).

\section{CONSIDERAÇÕES FINAIS}

Este estudo alcançou o objetivo de identificar e analisar fatores de risco para o suicídio que podem ser exacerbados pela situação de emergência sanitária global. Está claro que uma pandemia nas proporções inéditas da COVID-19 promove instabilidade psíquica e pode desencadear reações que potencializam o comportamento suicida, em seus mais variados graus. Nestes tempos sem precedentes, é inegável que a intensidade do sofrimento psíquico eleve a vulnerabilidade dos indivíduos para os agravos à saúde mental, inclusive o suicídio. Deve-se considerar a possibilidade de que as consequências adversas da pandemia sejam duradouras e se prolonguem para além da própria COVID-19.

No entanto, diversos autores, dentre os quais nos incluímos, concordam que essas previsões podem ter seus efeitos mitigados e muitas mortes podem ser prevenidas caso sejam implementadas estratégias nacionais consistentes e cientificamente fundamentadas de proteção à saúde mental (Brown \& Schuman, 2020; Gunnel et al., 2020; Petterson, 2020; Reger et al., 2020). Essas ações precisam alcançar prioridade na agenda da saúde pública, apoiadas inclusive por vigilância e colaboração no plano nacional e internacional (Gunnel et al., 2020). Nesse sentido, é fundamental que sejam promovidas estratégias multiprofissionais de prevenção, cuidado em saúde e posvenção que visem ao bem-estar das populações, com esforços que permitam fortalecer a esperança, equilibrar os sentimentos negativos e reinserir planos de vida (Ministério da Saúde, 2020).

A Organização Mundial da Saúde (OMS) reitera a importância do apoio e da adoção de soluções coordenadas por parte do governo, das autoridades locais, das lideranças dos setores público e privado para assegurar a sustentabilidade e continuidade de ações comunitárias que fortaleçam a coesão social e reduzam o sentimento de solidão e desamparo social, principalmente entre os mais vulneráveis, como os idosos (Nações Unidas Brasil, 2020b). São propostas estratégias compatíveis com as necessidades de manter o distanciamento social, como checagens regulares por telefone com pessoas que moram sozinhas e organização de atividades virtuais para estímulo intelectual e cognitivo, além do amparo sócio-afetivo.

Finalmente, é preciso destacar que começaram a emergir estudos com resultados divergentes, como o que aponta elevação nas taxas de suicídio entre jovens em diversos países, tais como Índia, Reino Unido e Estados Unidos (Manzar, Albougami, Usman, \& Mamun, 2021) e, por outro lado, dados que sugerem decréscimo no desejo de morrer por parte de pessoas que apresentavam risco de suicídio previamente à pandemia (Cobo et al., 2021). Isto mostra que os resultados 
não são conclusivos e que qualquer inferência definitiva acerca deste tema neste momento histórico é precipitada. É de suma importância que sejam conduzidos novos estudos que englobem diferentes contextos, países, populações e faixas etárias diversas.

A despeito dos inúmeros prejuízos e reveses psicológicos impostos pelos tempos difíceis de enfrentamento de uma pandemia com dimensões sem precedentes, é importante considerar que alguns fatores de proteção ao comportamento suicida também puderam ser identificados. São aspectos que merecem ser destacados na elaboração de um plano de intervenção psicológica que almeje o fortalecimento dos indivíduos e famílias afetadas, de modo a potencializar recursos que favoreçam a superação das sequelas das experiências traumáticas e facilitem a ressignificação dos diversos lutos individuais e coletivos.

\section{REFERÊNCIAS}

Alexandre, V., Vasconcelos, N. A. O., Santos, M. A., \& Monteiro, J. F. A. (2019). O acolhimento como postura na percepção de psicólogos hospitalares. Psicologia: Ciência e Profissão, 39, e188484, 1-14. https://doi.org/10.1590/1982-3703003188484

Arruda-Colli, M. N. F., Sansom-Daly, U., Santos, M. A., \& Wiener, L. (2018). Considerations for the cross-cultural adaptation of an advance care planning guide for youth with cancer. Clinical Practice in Pediatric Psychology, 6(4), 341-354. https://doi.org/10.1037/cpp0000254

Barbosa, C. G., Melchiori, L. E., \& Neme, C. M. B. (2011). O significado da morte para adolescentes, adultos e idosos. Paidéia (Ribeirão Preto), 21(49), 175-185. https://doi.org/10.1590/S0103$\underline{863 \times 2011000200005}$

Benites, A. C., Neme, C. M. B., \& Santos, M. A. (2017). Significados da espiritualidade para pacientes com câncer em cuidados paliativos. Estudos de Psicologia (Campinas), 34(2), 269-279. https://doi.org/10.1590/1982-02752017000200008

Bertolote, J. M., Mello-Santos, C., \& Botega, N. J. (2010). Detecção do risco de suicídio nos serviços de emergência psiquiátrica. Revista Brasileira de Psiquiatria, 32(Suppl. 2), S87-S95. https://doi.org/10.1590/S1516-44462010000600005

Bisby, A. (2020, june 16). New men's health studies reveal COVID silver lining: Stronger father-child connections. Canadian Men's Health Foundation. Recuperado de https://menshealthfoundation.ca/news/new-mens-health-studies-reveal-covid-silverlining-stronger-father-child-connections/

Borges, A. D. V. S., Silva, E. F., Toniollo, P. B., Mazer, S. M., Valle, E. R. M., \& Santos, M. A. (2006). Percepção da morte pelo paciente oncológico ao longo do desenvolvimento. Psicologia em Estudo (Maringá), 11(2), 361-369. https://doi.org/10.1590/S1413-73722006000200015

Botega, N. J. (2014). Comportamento suicida: Epidemiologia. Psicologia USP, 25(3), 231-236. https://doi.org/10.1590/0103-6564D20140004

Botega, N. J. (2015). Crise suicida: Avaliação e manejo. Porto Alegre, RS: Artmed.

Botega, N. J., Werlang, B. S. G., Cais, C. F. S., \& Macedo, M. M. K. (2006). Prevenção do comportamento suicida. Psico, 37(3), 213-220. Recuperado de http://revistaseletronicas.pucrs.br/ojs/index.php/revistapsico/article/view/1442/1130

Braga, I. F., Oliveira, W. A., \& Santos, M. A. (2020). História do presente de mulheres durante a pandemia da Covid-19: Feminização do cuidado e vulnerabilidade. Revista Feminismos, 8(3), 190-198. Recuperado de https://periodicos.ufba.br/index.php/feminismos/article/view/42459/23919

Brown, S., \& Schuman, D. L. (2020). Suicide in the time of COVID-19: A perfect storm. The Journal of Rural Health, 0, 1-4. https://doi.org/10.1111/jrh.12458

Cassorla, R. M. S. (2017). Suicídio - Fatores inconscientes e aspectos socioculturais: Uma introdução. São Paulo, SP: Blucher. 
Cardoso, E. A., Mastropietro A. P., Santos M. A., \& Voltarelli, J. C. (2010). Apoio psicológico na terminalidade: Ensinamentos para a vida. Psicologia em Estudo (Maringá), 15(1), 235-244. https://doi.org/10.1590/S1413-73722010000200002

Cobo, A., Porras-Segovia, A., Pérez-Rodríguez, M., Artés-Rodríguez, A., Barrigón, M., Courtet, P., \& Baca-García, E. (2021). Patients at high risk of suicide before and during a COVID-19 lockdown: Ecological momentary assessment study. BJPsych Open, 7, e82, 1-3. https://doi.org/10.1192/bjo.2021.43

Ferracioli, N. G. M., Oliveira-Cardoso, E. A., Vedana, K. G. G., Pillon, S. C., Miasso, A. I., Souza, J., Risk, E. N., Oliveira, W. A., Leonidas, C., \& Santos, M. A. (2019). Os bastidores psíquicos do suicídio: Uma compreensão psicanalítica. Vínculo, 16(1), 16-29. Recuperado de http://pepsic.bvsalud.org/scielo.php?script=sci_arttext\&pid =S1806-24902019000100003

Ferreira, H. G. (2021). Gender differences in mental health and beliefs about Covid-19 among elderly internet users. Paidéia (Ribeirão Preto), 31, e3110. https://doi.org/10.1590/1982-4327e3110

Ferreira Junior, A. (2015). O comportamento suicida no Brasil e no mundo. Revista Brasileira de Psicologia, 2(1), 15-28.

Freitas, I. S., Oliveira-Cardoso, E. A., \& Santos, M. A. (2017). Spirituality and religiosity in mothers of children with hematologic cancer. Psicologia em Estudo (Maringá), 22(3), 433-447. https://doi.org/10.4025/psicolestud.v22i3.34606

Fukumitsu, K. O, \& Kovács, M. J. (2015). O luto por suicídios: Uma tarefa da posvenção. Revista Brasileira de Psicologia, 2(2), 41-47.

Gil, A. C. (2002). Como elaborar projetos de pesquisa. São Paulo, SP: Atlas.

Grupe, D. W., \& Nitschke, J. B. (2013). Uncertainty and anticipation in anxiety: An integrated neurobiological and psychological perspective. Nature Reviews Neuroscience, 14(7), 488-501. https://doi.org/10.1038/nrn3524

Gunnell, D., Appleby, L., Arensman, E., Hawton, K., John, A., Kapur, N., Khan, M. . . Pirkis, J. (2020). Suicide risk and prevention during the COVID-19 pandemic. Lancet Psychiatry, 7(6), 468-471. https://doi.org/10.1016/S2215-0366(20)30171-1

Joiner, T. E. (2007). Why people die by suicide. U.S.A.: Harvard University Press

Manzar, M. D., Albougami, A., Usman, N., \& Mamun, M. A. (2021). Suicide among adolescents and youths during the COVID-19 pandemic lockdowns: A press media reports-based exploratory study. Journal of Child and Adolescent Psychiatric Nursing, 34(2), 139-146. https://doi.org/10.1111/jcap.12313

Mapa da Saúde Mental. Recuperado de https://mapasaudemental.com.br/atendimento-online-paraprofissionais-da-saude/

Ministério da Saúde (2020). Saúde mental e atenção psicossocial na pandemia COVID-19: Suicídio na pandemia COVID-19. Brasília, DF: Autor. Recuperado de https://www.arca.fiocruz.br/bitstream/icict/41420/2/Cartilha_PrevencaoSuicidioPandemia.p df

Nações Unidas Brasil. (2020, 14 de maio). OMS: O impacto da pandemia na saúde mental das pessoas já é extremamente preocupante. Recuperado de https://brasil.un.org/pt-br/85787-oms-oimpacto-da-pandemia-na-saude-mental-das-pessoas-ja-e-extremamente-preocupante

Oliveira-Cardoso, E. A., Silva, B. C. A., Santos, J. H., Lotério, L. S., Accoroni, A. G., \& Santos, M. A. (2020), The effect of suppressing funeral rituals during the COVID-19 pandemic on bereaved families. Revista Latino-Americana de Enfermagem, 28, e3361. https://doi.org/10.1590/15188345.4519 .3361

Oliveira, T. T. S. S., Fabrici, E. P., \& Santos, M. A. (2018). Estrutura e funcionamento de uma equipe de saúde mental de Trieste na perspectiva de seus integrantes: Um estudo qualitativo. Psicologia em Pesquisa, 12(2), 24-35. https://doi.org/10.24879/2018001200200139

Oliveira, W. A., Oliveira-Cardoso, E. A., Silva, J. L., \& Santos, M. A. (2020a). Impactos psicológicos e ocupacionais das sucessivas ondas recentes de pandemias em profissionais da saúde: Revisão integrativa e lições aprendidas. Estudos de Psicologia (Campinas), 37, e200066. https://doi.org/10.1590/1982-0275202037e200066

Oliveira, W., Magrin, J., Andrade, A., Micheli, D., Carlos, D., Fernandez, J., . . Santos, M. (2020b). Violência por parceiro íntimo em tempos da COVID-19: Scoping review. Psicologia, Saúde \& Doenças, 21(3), 606-623. http://dx.doi.org/10.15309/20psd210306

Oliveira, W. A., Silva, J. L., Andrade, A. L. M., Micheli, D., Fernández, J. E. R., Dellazzana-Zanon, L. L. . . Santos, M. A. (2020c). Adolescence in times of pandemic: Integrating consensus into a 
concept map. Estudos de Psicologia (Natal), 25(2), 133-143. http://dx.doi.org/10.22491/1678-4669.20200014

Parkes, C. M. (1998). Luto: Estudos sobre a perda na vida adulta (M. H. F. Bromberg, Trad.). São Paulo, SP: Summus.

Pereira, A., \& Cardoso, F. (2015). Suicidal ideation in university students: Prevalence and association with school and gender. Paidéia (Ribeirão Preto), 25(62), 299-306. https://doi.org/10.1590/1982-43272562201503

Petterson, S., Westfall, J. M., \& Miller, B. F. (2020). Projected deaths of despair during the coronavirus recession. U.S.A.: Well Being Trust. Recuperado de https://www.grahamcenter.org/content/dam/rgc/documents/publications-reports/reports/Projected-DeathsDespair-COVID-19.pdf

Prado, A. S., \& Freitas, J. L. (2020). Social distancing during pandemics: Suicide risk and prevention in the face of psychosocial impacts of COVID-19. Estudos de Psicologia (Natal), 25(2), 157-166. http://dx.doi.org/10.22491/1678-4669.20200016

Reger, M. A., Stanley, I. H. \& Joiner, T. E. (2020). Suicide mortality and coronavirus disease 2019: A $\begin{array}{llll}\text { perfect storm? } & \text { 1093-1094 }\end{array}$ https://doi.org/10.1001/jamapsychiatry.2020.1060

Rezende, J. M. (1998). Epidemia, endemia, pandemia, epidemiologia. Revista de Patologia Tropical. 27(1), 153-155. Recuperado de https://www.revistas.ufg.br/iptsp/article/view/17199/10371

Rother, E. T. (2007). Revisão sistemática X revisão narrativa. Acta Paulista de Enfermagem, 20(2), v-vi. https://doi.org/10.1590/S0103-21002007000200001

Sakamoto, L. (2020, 31 de maio). Atendimento do SAMU relacionado a suicídio cresce durante a pandemia. Notícias.uol.com. Recuperado de https://noticias.uol.com.br/colunas/leonardosakamoto/2020/05/31/atendimento-de-urgencia-relacionado-a-suicidio-cresce-durante-apandemia.htm

Santos, M. A. (2017). Câncer e suicídio em idosos: Determinantes psicossociais do risco, psicopatologia e oportunidades para prevenção. Ciência \& Saúde Coletiva, 22(9), 3061-3075. https://doi.org/10.1590/1413-81232017229.05882016

Santos, M. A., \& Hormanez, M. (2013). Atitude frente à morte em profissionais e estudantes de enfermagem: Revisão da produção científica da última década. Ciência \& Saúde Coletiva, 18(9), 2757-2768. https://doi.org/10.1590/S1413-81232013000900031

Santos, M. A., Aoki, F. C. O. S., \& Oliveira-Cardoso, E. A. (2013). Significado da morte para médicos frente à situação de terminalidade de pacientes submetidos ao Transplante de Medula Óssea. Ciência \& Saúde Coletiva, 18(9), 2625-2634. https://doi.org/10.1590/S141381232013000900017

Santos, M. A., Oliveira, W. A., \& Oliveira-Cardoso, E. A. (2020). Inconfidências de abril: Impacto do isolamento social na comunidade trans em tempos de pandemia de COVID-19. Psicologia \& Sociedade, 32, e020018. https://doi.org/10.1590/1807-0310/2020v32240339

Schmidt, B., Gabarra, L. M., \& Gonçalves, J. R. (2011). Intervenção psicológica em terminalidade e morte: relato de experiência. Paidéia (Ribeirão Preto), 21(50), 423-430. https://doi.org/10.1590/S0103-863X2011000300015

Scorsolini-Comin, F., Rossato, L., \& Santos, M. A. (2020). Saúde mental, experiência e cuidados: Implicações da pandemia de COVID-19. Revista da SPAGESP, 21(2), 1-6. Recuperado de http://pepsic.bvsalud.org/scielo.php?script=sci_arttext\&pid=S1677$29702020000200001 \& \operatorname{lng}=p t \& t \operatorname{lng}=\mathrm{pt}$

Siqueira, H. B. O. M., Gomez, R. R. F., Saltareli, S., Silva, T. C. R., Silva, C. L., Santos, M. A., \& Sousa, F. A. E. F. (2019). A dor da morte em famílias de crianças com câncer: Revisão integrativa de literatura. Saúde Coletiva, 9(50), 1736-1744. Recuperado de http://revistas.mpmcomunicacao.com.br/index.php/saudecoletiva/article/view/149/144.

Soares, C. F. S., Araújo, R. S., Estrela, F. M., Morais, A. C., Farias, R. V., Almeida, V. R. S., . . Cruz, N. R. (2020). Fatores precipitantes e/ou agravantes da violência contra crianças no contexto da COVID-19. Brazilian Journal of Development, $7(1), \quad 9430-9442$ https://doi.org/10.34117/bjdv7n1-638

Teixeira, C. F. S., Soares, C. M., Souza, E. A., Lisbos, E. S., Pinto, I. C. M., Andrade, L. R., \& Espiridião, M. A. (2020). A saúde dos profissionais de saúde no enfrentamento da pandemia de Covid-19. Ciência \& Saúde Coletiva, 25(9), 3465-3474. https://doi.org/10.1590/141381232020259.19562020

Vattimo, E. F. Q. (2020). A pandemia e as liberdades individuais. Ser Médico, 90, 32-33. 
Vedana, G. V., Magrini, D. F., Zanetti, A. C. G., Miasso, A., Borges, T. L., \& Santos, M. A. (2017). Attitudes towards suicidal behaviour and associated factors among nursing professionals: A quantitative study. Journal of Psychiatric and Mental Health Nursing. 24(9-10), 651-659. https://doi.org/10.1111/jpm.12413

Weissbourd, R., Batanova, M., McIntyre, J., \& Torres, E. (2020). How the pandemic is strengthening fathers' relationships with their children. Cambridge: Harvard School of Education. Recuperado de https://mcc.gse.harvard.edu/

Werneck, G. L., \& Carvalho, M. S. (2020). A pandemia de COVID-19 no Brasil: crônica de uma crise sanitária anunciada. Cadernos de Saúde Pública, 36(5), e00068820. https://doi.org/10.1590/0102-311X00068820

WHO, World Health Organization. (2019). Suicide. Geneva: World Health Organization. Recuperado de https://www.who.int/news-room/fact-sheets/detail/suicide

WHO, World Health Organization. (2021). Weekly operational update on COVID-19 - 28 June 2021. Geneva: World Health Organization. Recuperado de https://www.who.int/emergencies/diseases/novel-coronavirus-2019/situation-reports

\section{AGRADECIMENTOS}

Os autores agradecem ao Conselho Nacional de Desenvolvimento Científico e Tecnológico - Brasil (CNPq) pelo financiamento da pesquisa e à equipe da Inclinare Psicologia pelo apoio e inspiração.

\section{CONFLITOS DE INTERESSES}

Os autores declararam que não houve conflitos de interesses para realização desta pesquisa.

\section{FINANCIAMENTO}

Conselho Nacional de Desenvolvimento Científico e Tecnológico - Brasil (CNPq).

\section{SOBRE OS AUTORES}

Natália Gallo Mendes Ferracioli é psicóloga e mestranda pela Faculdade de Filosofia, Ciências e Letras de Ribeirão Preto da Universidade de São Paulo (FFCLRP-USP). Possui especialização em saúde mental e em Psicologia Hospitalar, com experiência em implantação e coordenação de serviços de Psicologia. Membro do Laboratório de Ensino e Pesquisa em Psicologia da Saúde - LEPPS (FFCLRPUSP-CNPq). Fundadora da Inclinare Psicologia e coordenadora da Pós-Graduação em Psicologia Hospitalar e da Saúde do Centro Universitário Barão de Mauá, Ribeirão Preto-SP. Suas pesquisas concentram-se no campo da Psicologia Hospitalar e da Saúde, em suas interfaces: enfrentamento do processo saúde-doença, suicídio, psicologia clínica e psicanálise.

E-mail: nataliagmendes@hotmail.com

(2) https://orcid.org/0000-0003-3912-1531.

Wanderlei Abadio de Oliveira é Professor Permanente do Programa de Pós-Graduação Stricto Sensu em Psicologia da Pontifícia Universidade Católica de Campinas (PUC-Campinas). É psicólogo, Doutor em Ciências (Doutorado Direto) pela Universidade de São Paulo (USP), em cotutela com a Scuola di Dottorato di Ricerca in Psicologia da Università Cattolica del Sacro Cuore (Milão-Itália). Pósdoutorado pelo Departamento de Psicologia da Faculdade de Filosofia, Ciências e Letras de Ribeirão Preto (FFCLRP-USP), como bolsista do Programa Nacional de Pós-Doutorado PNPD/CAPES. Editor Associado e membro da Comissão Editorial do periódico Paidéia (Ribeirão Preto), Qualis Capes A1. Pesquisador associado do Laboratório de Ensino e Pesquisa em Psicologia da Saúde - LEPPS (FFCLRPUSP-CNPq). Suas pesquisas concentram-se no campo da Psicologia do Desenvolvimento em suas interfaces: bullying, violência escolar, desengajamento moral, família, saúde da criança e do adolescente, e nos campos da psicologia social, saúde coletiva, políticas públicas e determinantes sociais da saúde.

E-mail: wanderleio@hotmail.com

https://orcid.org/0000-0002-3146-8197. 
Érika Arantes de Oliveira-Cardoso é Professora Doutora do Programa de Pós-Graduação em Psicologia da Faculdade de Filosofia, Ciências e Letras de Ribeirão Preto da Universidade de São Paulo (FFCLRP-USP). Psicóloga, mestre e doutora pelo Programa de Pós-Graduação em Psicologia da FFCLRP-USP. Especialista em Psicologia Hospitalar (Conselho Federal de Psicologia). Vicecoordenadora do Laboratório de Ensino e Pesquisa em Psicologia da Saúde - LEPPS (FFCLRP-USPCNPq). Coordenadora do Laboratório Lutos e Terminalidades - LUTE (FFCLRP-USP-CNPq). Trabalha no campo da Psicologia da Saúde, nas interfaces enfrentamento do processo saúde-doença, diversidade de orientações sexuais e identidades de gênero.

E-mail: erikaao@ffclrp.usp.br

(6) http://orcid.org/0000-0001-7986-0158.

Clarissa Mendonça Corradi-Webster é Professora Associada do Programa de Pós-Graduação em Psicologia da Faculdade de Filosofia, Ciências e Letras de Ribeirão Preto da Universidade de São Paulo (FFCLRP-USP). Psicóloga, mestre pelo Programa de Pós-Graduação em Saúde na Comunidade da Faculdade de Medicina de Ribeirão Preto - USP e doutora pelo Programa de Pós-Graduação em Psicologia da FFCLRP-USP. Coordenadora do Laboratório de Ensino e Pesquisa em Psicopatologia, Drogas e Sociedade - LePsis (FFCLRP-USP). Editora Associada e membro da Comissão Editorial do periódico Paidéia (Ribeirão Preto). Trabalha no campo da Psicologia da Saúde nas interfaces com saúde mental, uso de álcool e outras drogas.

E-mail: clarissac@usp.br

(2) https://orcid.org/0000-0002-3386-1267.

Eduardo Name Risk é servidor público federal, Professor Adjunto do Departamento de Psicologia do Centro de Educação e Ciências Humanas $(\mathrm{CECH})$ da Universidade Federal de São Carlos (UFSCar). Psicólogo e Bacharel em Psicologia pela Faculdade de Filosofia, Ciências e Letras de Ribeirão Preto (FFCLRP) da Universidade de São Paulo (USP), Mestre em Psicologia (Área de concentração Psicologia) e Doutor em Psicologia (Área de concentração - Psicologia em Saúde e Desenvolvimento) pela FFCLRP-USP. Realizou estágio de pós-doutorado no Departamento de Psicologia da FFCLRPUSP com bolsa de Pós-Doutorado Júnior (PDJ) do Conselho Nacional de Desenvolvimento Científico e Tecnológico - Brasil (CNPq). Membro do Laboratório de Ensino e Pesquisa em Psicologia da Saúde - LEPPS (FFCLRP-USP-CNPq). Editor Adjunto do periódico Paidéia (Ribeirão Preto), Editor Associado do periódico Cadernos de Psicologia (Sociedade Brasileira de Psicologia - SBP). Trabalha no campo da Psicologia nas interfaces com a Psicanálise e com a Sociologia.

E-mail: eduardorisk@ufscar.br

\section{(1) https://orcid.org/0000-0001-7290-2597.}

Manoel Antônio dos Santos é Professor Titular do Departamento de Psicologia e do Programa de Pós-Graduação em Psicologia da Faculdade de Filosofia, Ciências e Letras de Ribeirão Preto da Universidade de São Paulo (FFCLRP-USP). Bolsista de Produtividade em Pesquisa do CNPq nível $1 \mathrm{~A}$. Psicólogo pelo Instituto de Psicologia da Universidade de São Paulo (IPUSP). Mestre e Doutor pelo Programa de Pós-Graduação em Psicologia Clínica do IPUSP. Livre-docente em Psicoterapia Psicanalítica pela FFCLPR-USP. Especialista em Terapia Familiar e de Casal pelo Instituto Familae (Ribeirão Preto); especialista em Psicologia Clínica e Psicologia Hospitalar (Conselho Federal de Psicologia). Coordenador do Laboratório de Ensino e Pesquisa em Psicologia da Saúde - LEPPS (FFCLRP-USP-CNPq). Membro Titular da Academia Paulista de Psicologia, cadeira 33. Editor Chefe do periódico Paidéia (Ribeirão Preto). Suas pesquisas concentram-se no campo da Psicologia da Saúde, nas interfaces enfrentamento do processo saúde-doença, diversidade de orientações sexuais e identidades de gênero.

E-mail: masantos@ffclrp.usp.br

(1) https://orcid.org/0000-0001-8214-7767. 